\title{
Resolution in the Case of the Hydrogen Atom of an Improved Dirac Equation
}

\author{
Claude Daviau', Jacques Bertrand², Raymond Albert Ng ${ }^{3}$ \\ ${ }^{1}$ Le Moulin de la Lande, Pouillé-les-Côteaux, France \\ ${ }^{2} 15$ Avenue Danielle Casanova, Saint-Gratien, France \\ ${ }^{3}$ De La Salle University (DLSU), Manila, Philippines \\ Email: daviau.claude@orange.fr, bertrandjacques-m@orange.fr, raymondalbertng@gmail.com
}

How to cite this paper: Daviau, C., Bertrand, J. and Ng, R.A. (2020) Resolution in the Case of the Hydrogen Atom of an Improved Dirac Equation. Journal of Modern Physics, 11, 1075-1090. https://doi.org/10.4236/jmp.2020.117068

Received: June 11, 2020

Accepted: July 17, 2020

Published: July 20, 2020

Copyright $\odot 2020$ by author(s) and Scientific Research Publishing Inc. This work is licensed under the Creative Commons Attribution International License (CC BY 4.0).

http://creativecommons.org/licenses/by/4.0/

\begin{abstract}
The improved Dirac equation is completely solved in the case of the hydrogen atom. A method of separation of variables in spherical coordinates is used. The angular functions are the same as with the linear Dirac equation: they account for the spin $1 / 2$ of the electron. The existence of a probability density governs the radial equations. This gives all the quantum numbers required by spectroscopy, the true number of energy levels and the true levels obtained by Sommerfeld's formula.
\end{abstract}

\section{Keywords}

Electromagnetism, Clifford Algebra, Dirac Equation, Lagrangian Formalism, Hydrogen Atom

\section{Introduction}

The improved Dirac equation was obtained from Lochak's theory of a leptonic magnetic monopole [1] [2] [3]. In this theory, the quantum wave has two $U(1)$ gauge invariances. The mass term of the leptonic magnetic monopole is then able to replace the usual mass term of the Dirac equation. First, the non-linear mass term was read in the frame of Hestenes's space-time algebra [4] [5] [6]-[11]. The improved equation may be obtained from the simplification of the Lagrangian density of the Dirac equation, where the $m \bar{\psi} \psi=m \cos (\beta) \rho$ mass term is replaced by $m \rho$. The Dirac wave of the linear equation or of the improved equation may be read as functions of space-time with values in the Clifford algebra $\mathrm{Cl}_{3}$ [12]-[34].

Since 1928, the relativistic invariance of Dirac's theory used the previous Pauli matrices for the spin of the electron: the space-time variable $x=\left(x^{0}, x^{1}, x^{2}, x^{3}\right)$ 
was replaced by

$$
x=x^{0}+\vec{x}=\left(\begin{array}{cc}
x^{0}+x^{3} & x^{1}-i x^{2} \\
x^{1}+i x^{2} & x^{0}-x^{3}
\end{array}\right), x^{0}=c t .
$$

This is equivalent to saying that the three Pauli matrices:

$$
\sigma_{1}=\left(\begin{array}{cc}
0 & 1 \\
1 & 0
\end{array}\right) ; \sigma_{2}=\left(\begin{array}{cc}
0 & -i \\
i & 0
\end{array}\right) ; \sigma_{3}=\left(\begin{array}{cc}
1 & 0 \\
0 & -1
\end{array}\right) \text {, }
$$

form a orthogonal oriented basis in space. We shall put arrows on vectors in space, so any vector reads

$$
\vec{v}=v^{j} \sigma_{j}=v^{1} \sigma_{1}+v^{2} \sigma_{2}+v^{3} \sigma_{3} .
$$

The geometric algebra of space $\mathrm{Cl}_{3}$ and $M_{2}(\mathbb{C})$ are isomorphic algebras on the real field; the sum and the product of matrices are familiar in quantum physics. This matrix representation identifies complex numbers and scalar matrices in the Pauli algebra. With this identification, we write the $x$ of (1) as $x=x^{\mu} \sigma_{\mu}$, we consider $\left(\sigma_{0}, \sigma_{1}, \sigma_{2}, \sigma_{3}\right)$ as a basis in space-time and we use the Einstein summation convention, with superscripts and subscripts, where Latin indices are in space and Greek indices in space-time. Any element $z$ in the Clifford algebra of space $\mathrm{Cl}_{3}$ is a sum of a real part $x$, a vector part $\vec{v}$, an axial-vector part $i \vec{w}$ and a pseudo-scalar part $i y$, and we need:

$$
\begin{aligned}
& z=x+\vec{v}+i \vec{w}+i y ; \hat{z}=x-\vec{v}+i \vec{w}-i y, \\
& \tilde{z}=z^{\dagger}=x+\vec{v}-i \vec{w}-i y ; \bar{z}=x-\vec{v}-i \vec{w}+i y .
\end{aligned}
$$

The application $z \mapsto \hat{z}$ is the main automorphism of $\mathrm{Cl}_{3}$. The reverse is also the adjoint (transposed conjugate matrix), so $z \mapsto \tilde{z}=z^{\dagger}$ is the reversion. The third conjugation, $z \mapsto \bar{z}$ is the product of the two previous ones and we shall need:

$$
\bar{z}=\hat{z}^{\dagger}=\widehat{z^{\dagger}} ; \widehat{A B}=\hat{A} \hat{B} ; \overline{A B}=\bar{B} \bar{A} ; M \bar{M}=\bar{M} M=\operatorname{det}(M)
$$

Space-time is then made of the auto-adjoint part of the space algebra. We use:

$$
\begin{gathered}
\hat{x}=\bar{x}=x^{0}-\vec{x} ; x^{\dagger}=x, \\
\operatorname{det}(x)=x \hat{x}=x \cdot x=\left(x^{0}\right)^{2}-\vec{x}^{2}=\left(x^{0}\right)^{2}-\left(x^{1}\right)^{2}-\left(x^{2}\right)^{2}-\left(x^{3}\right)^{2} .
\end{gathered}
$$

The main reason to use the geometric algebra $\mathrm{Cl}_{3}$ is the ability to read all relativistic quantum physics in this algebra: the electron wave is a function of space and time in $\mathrm{Cl}_{3}$ :

$$
x \mapsto \phi=\phi(x) \in C l_{3} .
$$

The link between $\mathrm{Cl}_{3}$ and the complex formalism is simple only if we use the left and right Weyl spinors $\eta$ and $\xi$, by letting:

$$
\begin{aligned}
& \phi=\sqrt{2}\left(\begin{array}{ll}
\xi & \hat{\eta}
\end{array}\right)=\sqrt{2}\left(\begin{array}{cc}
\xi_{1} & -\bar{\eta}_{2} \\
\xi & \bar{\eta}_{1}
\end{array}\right) ; \hat{\eta}=\left(\begin{array}{c}
-\bar{\eta}_{2} \\
\bar{\eta}_{1}
\end{array}\right), \\
& \hat{\phi}=\sqrt{2}(\eta \hat{\xi})=\sqrt{2}\left(\begin{array}{cc}
\eta_{1} & -\bar{\xi}_{2} \\
\eta_{2} & \bar{\xi}_{1}
\end{array}\right) ; \hat{\xi}=\left(\begin{array}{c}
-\bar{\xi}_{2} \\
\bar{\xi}_{1}
\end{array}\right) .
\end{aligned}
$$


The usual formalism uses a $\psi$ and $\gamma^{\mu}$ matrices defined as following:

$$
\begin{gathered}
\psi=\left(\begin{array}{l}
\xi \\
\eta
\end{array}\right) ; \gamma^{0}=\gamma_{0}=\left(\begin{array}{cc}
0 & I \\
I & 0
\end{array}\right) ; I=\sigma^{0}=\sigma_{0}=\left(\begin{array}{ll}
1 & 0 \\
0 & 1
\end{array}\right), \\
\gamma^{j}=-\gamma_{j}=\left(\begin{array}{cc}
0 & -\sigma_{j} \\
\sigma_{j} & 0
\end{array}\right), j=1,2,3 .
\end{gathered}
$$

Our improved wave equation of the electron, which has the Dirac equation as linear approximation, reads [6]-[19]:

$$
\bar{\phi}(\nabla \hat{\phi}) \sigma_{21}+\bar{\phi} q A \hat{\phi}+m \rho=0 ; \nabla=\sigma^{\mu} \partial_{\mu} ; \sigma_{21}=\sigma_{2} \sigma_{1} ; \rho=|\operatorname{det}(\phi)| .
$$

where $q=e / \hbar c, \quad m=m_{0} c / \hbar, \quad \sigma^{0}=\sigma_{0}, \sigma^{j}=-\sigma_{j}, j=1,2,3$. This equation is invariant under any transformation $D$ defined by an element $M$ of the Lie group $\mathrm{Cl}_{3}^{*}$ (group of invertible elements of $\mathrm{Cl}_{3}$ ):

$$
\begin{gathered}
x^{\prime}=D(x)=M x M^{\dagger}=x^{\prime \mu} \sigma_{\mu} ; \partial_{\mu}^{\prime}=\frac{\partial}{\partial x^{\prime \mu}} ; x^{\prime v}=D_{\mu}^{v} x^{\mu}, \\
\nabla=\bar{M} \nabla^{\prime} \hat{M} ; \nabla^{\prime}=\sigma^{\mu} \partial_{\mu}^{\prime} ; q A=\bar{M} q^{\prime} A^{\prime} \hat{M} \\
\phi^{\prime}\left(x^{\prime}\right)=M \phi(x) ; \xi^{\prime}=M \xi ; \eta^{\prime}=\hat{M} \eta .
\end{gathered}
$$

Multiplying (11) on the left side by $\bar{\phi}^{-1}$, we obtain the usual form in the Pauli algebra of the improved equation:

$$
\nabla \hat{\phi} \sigma_{21}+q A \hat{\phi}+m \mathrm{e}^{-i \beta} \phi=0 ; \operatorname{det}(\phi)=\rho \mathrm{e}^{i \beta},
$$

where $\beta$ is the Yvon-Takabayasi angle. In the usual formalism of complex $4 \times 4$ matrices, much more complicated than the Pauli algebra, this wave equation reads:

$$
0=\left[\gamma^{\mu}\left(\partial_{\mu}+i q A_{\mu}\right)+i m \exp \left(-\beta \gamma_{0} \gamma_{1} \gamma_{2} \gamma_{3}\right)\right] \psi
$$

Our wave equation has the Dirac equation as linear approximation: if $\beta$ is null or negligible, the improved Equation (15) is reduced to the Dirac equation that reads in the Pauli algebra and in the Dirac algebra:

$$
\begin{gathered}
0=\nabla \hat{\phi} \sigma_{21}+q A \hat{\phi}+m \phi, \\
0=\left[\gamma^{\mu}\left(\partial_{\mu}+i q A_{\mu}\right)+i m\right] \psi .
\end{gathered}
$$

Our equation is an improvement from the linear Dirac equation for many reasons explained in our previous papers [6]-[34]: Charge conjugation gives a positron with positive mass-energy. The gauge group comprises a local electric gauge linked to the conservative current of probability and a second gauge (only global) that is the chiral gauge of the Yvon-Takabayasi angle, linked to a second conservative current. This $U(1)$ gauge invariance corresponds to the first part of the $U(1) \times S U(2)$ group of electro-weak interactions. Therefore, our improved equation allows us a generalization, with mass term, to all fermion waves of leptons and quarks. In this paper, we shall show how this improved, non-linear wave equation is nevertheless able to obtain the following results: the 
true quantum numbers, the true number of energy levels and the true energy levels in the case of the hydrogen atom. This was previously never obtained from a non-linear wave equation; therefore, everyone was thinking that the linearity of quantum mechanics was necessary, even for the linking to the non-linear relativistic gravitation.

\section{Separating Variables}

To solve the Dirac equation or the improved equation in the case of the hydrogen atom, two methods exist. We shall use here, not the initial method based on the non-relativistic approximation of the wave equation, but the new method invented by $\mathrm{H}$. Krüger [35], separating the variables in spherical coordinates:

$$
x^{1}=r \sin \theta \cos \varphi ; x^{2}=r \sin \theta \sin \varphi ; x^{3}=r \cos \theta .
$$

We use the following notation:

$$
\begin{gathered}
i_{1}=\sigma_{23}=i \sigma_{1} ; i_{2}=\sigma_{31}=i \sigma_{2} ; i_{3}=\sigma_{12}=i \sigma_{3}, \\
S=\mathrm{e}^{-\frac{\phi}{2} i_{3}} \mathrm{e}^{-\frac{\theta}{2} i_{2}} ; \Omega=\hat{\Omega}=r^{-1}(\sin \theta)^{-\frac{1}{2}} S, \\
\vec{\partial}=\sigma_{1} \partial_{1}+\sigma_{2} \partial_{2}+\sigma_{3} \partial_{3} ; \vec{\partial}^{\prime}=\sigma_{3} \partial_{r}+\frac{1}{r} \sigma_{1} \partial_{\theta}+\frac{1}{r \sin \theta} \sigma_{2} \partial_{\varphi} .
\end{gathered}
$$

H. Krüger obtained the remarkable identity:

$$
\vec{\partial}=\Omega \vec{\partial}^{\prime} \Omega^{-1},
$$

which with

$$
\nabla^{\prime}=\partial_{0}-\vec{\partial}^{\prime}=\partial_{0}-\left(\sigma_{3} \partial_{r}+\frac{1}{r} \sigma_{1} \partial_{\theta}+\frac{1}{r \sin \theta} \sigma_{2} \partial_{\varphi}\right)
$$

gives also:

$$
\Omega^{-1} \nabla=\nabla^{\prime} \Omega^{-1}
$$

Aiming for the separation of the temporal variable $x^{0}=c t$ and the angular variable $\varphi$ from the radial variable $r$ and the other angular variable $\theta$, we let:

$$
\phi=\Omega X \mathrm{e}^{\left(\lambda \varphi-E x^{0}+\delta\right) i_{3}} ; X=(\xi \hat{\eta}),
$$

where $X$ is a function (with value in the Pauli algebra) of only $r$ and $\theta, \hbar c E$ is the energy of the electron, $\delta$ is an arbitrary phase (that plays no role here because the wave equations are electric gauge-invariant), and $\lambda$ is a real constant which will be interpreted as the magnetic quantum number (we name here this quantum number $\lambda$ because $m$ is used in the mass term). We then get:

$$
\begin{aligned}
& \Omega^{-1} \phi=X \mathrm{e}^{\left(\lambda \varphi-E x^{0}+\delta\right) i_{3}}, \\
& \Omega^{-1} \hat{\phi}=\hat{X} \mathrm{e}^{\left(\lambda \varphi-E x^{0}+\delta\right) i_{3}} .
\end{aligned}
$$

We also have:

$$
\rho \mathrm{e}^{i \beta}=\operatorname{det}(\phi)=\operatorname{det}(\Omega) \operatorname{det}(X) \operatorname{det}\left[\mathrm{e}^{\left(\lambda \varphi-E x^{0}+\delta\right) i_{3}}\right],
$$




$$
\begin{gathered}
\operatorname{det}(\Omega)=r^{-2}(\sin \theta)^{-1} ; \operatorname{det}\left[\mathrm{e}^{\left(\lambda \varphi-E x^{0}+\delta\right) i_{3}}\right]=1, \\
\rho \mathrm{e}^{i \beta}=\frac{\operatorname{det}(X)}{r^{2} \sin \theta} .
\end{gathered}
$$

Then if we let

$$
\rho_{X} \mathrm{e}^{i \beta_{X}}=\operatorname{det}(X)
$$

we get:

$$
\rho=\frac{\rho_{X}}{r^{2} \sin \theta} ; \beta=\beta_{X} .
$$

Then with the form (26) of the wave, the Yvon-Takabayasi angle $\beta$ depends neither on the time nor on the $\varphi$ angle. It depends only on $r$ and $\theta$. Hence the separation of variables can be similarly obtained for the Dirac equation or for the improved equation. We have:

$$
\begin{aligned}
\nabla^{\prime} \Omega^{-1} \hat{\phi}=\left(\partial_{0}-\right. & \left.\sigma_{3} \partial_{r}-\frac{1}{r} \sigma_{1} \partial_{\theta}-\frac{1}{r \sin \theta} \sigma_{2} \partial_{\varphi}\right)\left[\hat{X} \mathrm{e}^{\left(\lambda \varphi-E x^{0}+\delta\right)_{3}}\right] \\
\partial_{0}\left(\hat{X} \mathrm{e}^{\left(\lambda \varphi-E x^{0}+\delta\right)_{3}}\right) & =-E \hat{X} i_{3} \mathrm{e}^{\left(\lambda \varphi-E x^{0}+\delta\right)_{i_{3}}} \\
\partial_{r}\left(\hat{X} \mathrm{e}^{\left(\lambda \varphi-E x^{0}+\delta\right) i_{3}}\right) & =\left(\partial_{r} \hat{X}\right) \mathrm{e}^{\left(\lambda \varphi-E x^{0}+\delta\right)_{3}} \\
\partial_{\theta}\left(\hat{X} \mathrm{e}^{\left(\lambda \varphi-E x^{0}+\delta\right) i_{3}}\right) & =\left(\partial_{\theta} \hat{X}\right) \mathrm{e}^{\left(\lambda \varphi-E x^{0}+\delta\right) i_{3}} \\
\partial_{\varphi}\left(\hat{X} \mathrm{e}^{\left(\lambda \varphi-E x^{0}+\delta\right) i_{3}}\right) & =\lambda \hat{X} i_{3} \mathrm{e}^{\left(\lambda \varphi-E x^{0}+\delta\right) i_{3}}
\end{aligned}
$$

We then get:

$$
\nabla \hat{\phi}=\Omega\left(-E \hat{X} i_{3}-\sigma_{3} \partial_{r} \hat{X}-\frac{1}{r} \sigma_{1} \partial_{\theta} \hat{X}-\frac{\lambda}{r \sin \theta} \sigma_{2} \hat{X} i_{3}\right) \mathrm{e}^{\left(\lambda \varphi-E x^{0}+\delta\right) i_{3}} .
$$

For the hydrogen atom we have:

$$
q A=q A^{0}=-\frac{\alpha}{r} ; \alpha=\frac{\mathrm{e}^{2}}{\hbar c},
$$

where $\alpha$ is the fine structure constant. We have:

$$
q A \hat{\phi} \sigma_{12}=-\frac{\alpha}{r} \hat{\phi} i_{3}=-\frac{\alpha}{r} \Omega \hat{X} \mathrm{e}^{\left(\lambda \varphi-E x^{0}+\delta\right) i_{3}} i_{3}=\Omega\left(-\frac{\alpha}{r} \hat{X} i_{3}\right) \mathrm{e}^{\left(\lambda \varphi-E x^{0}+\delta\right) i_{3}} .
$$

Also the wave Equation (15) becomes:

$$
-E \hat{X} i_{3}-\sigma_{3} \partial_{r} \hat{X}-\frac{1}{r} \sigma_{1} \partial_{\theta} \hat{X}-\frac{\lambda}{r \sin \theta} \sigma_{2} \hat{X} i_{3}-\frac{\alpha}{r} \hat{X} i_{3}+m \mathrm{e}^{-i \beta} X i_{3}=0,
$$

which means:

$$
\left(E+\frac{\alpha}{r}\right) \hat{X} i_{3}+\sigma_{3} \partial_{r} \hat{X}+\frac{1}{r} \sigma_{1} \partial_{\theta} \hat{X}+\frac{\lambda}{r \sin \theta} \sigma_{2} \hat{X} i_{3}=m \mathrm{e}^{-i \beta} X i_{3},
$$

The Dirac equation, in contrast, gives: 


$$
\left(E+\frac{\alpha}{r}\right) \hat{X} i_{3}+\sigma_{3} \partial_{r} \hat{X}+\frac{1}{r} \sigma_{1} \partial_{\theta} \hat{X}+\frac{\lambda}{r \sin \theta} \sigma_{2} \hat{X} i_{3}=m X i_{3} .
$$

Now we let, $\bar{z}$ being the complex conjugate of $z$.

$$
X=\left(\begin{array}{cc}
\mathbf{a} & -\overline{\mathbf{b}} \\
\mathbf{c} & \overline{\mathbf{d}}
\end{array}\right),
$$

where $\mathbf{a}, \mathbf{b}, \mathbf{c}, \mathbf{d}$ are functions with complex values of the real variables $r$ and $\theta$. We get:

$$
\hat{X}=\left(\begin{array}{cc}
\mathbf{d} & -\overline{\mathbf{c}} \\
\mathbf{b} & \overline{\mathbf{a}} .
\end{array}\right) .
$$

We then obtain the following equations:

$$
\begin{gathered}
i m \mathrm{e}^{-i \beta} X \sigma_{3}=i \mathrm{e}^{-i \beta}\left(\begin{array}{cc}
m \mathbf{a} & m \overline{\mathbf{b}} \\
m \mathbf{c} & -m \overline{\mathbf{d}}
\end{array}\right), \\
\hat{X} i_{3}=\left(\begin{array}{cc}
\mathbf{d} & -\overline{\mathbf{c}} \\
\mathbf{b} & \overline{\mathbf{a}}
\end{array}\right)\left(\begin{array}{cc}
i & 0 \\
0 & -i
\end{array}\right)=\left(\begin{array}{cc}
i \mathbf{d} & i \overline{\mathbf{c}} \\
i \mathbf{b} & -i \overline{\mathbf{a}}
\end{array}\right), \\
\sigma_{3} \partial_{r} \hat{X}=\left(\begin{array}{cc}
1 & 0 \\
0 & -1
\end{array}\right)\left(\begin{array}{cc}
\partial_{r} \mathbf{d} & -\partial_{r} \overline{\mathbf{c}} \\
\partial_{r} \mathbf{b} & \partial_{r} \overline{\mathbf{a}}
\end{array}\right)=\left(\begin{array}{cc}
\partial_{r} \mathbf{d} & -\partial_{r} \overline{\mathbf{c}} \\
-\partial_{r} \mathbf{b} & -\partial_{r} \overline{\mathbf{a}}
\end{array}\right), \\
\sigma_{1} \partial_{\theta} \hat{X}=\left(\begin{array}{cc}
0 & 1 \\
1 & 0
\end{array}\right)\left(\begin{array}{cc}
\partial_{\theta} \mathbf{d} & -\partial_{\theta} \overline{\mathbf{c}} \\
\partial_{\theta} \mathbf{b} & \partial_{\theta} \overline{\mathbf{a}}
\end{array}\right)=\left(\begin{array}{cc}
\partial_{\theta} \mathbf{b} & \partial_{\theta} \overline{\mathbf{a}} \\
\partial_{\theta} \mathbf{d} & -\partial_{\theta} \overline{\mathbf{c}}
\end{array}\right), \\
\sigma_{2} \hat{X} i_{3}=i_{2} \hat{X} \sigma_{3}=\left(\begin{array}{cc}
0 & 1 \\
-1 & 0
\end{array}\right)\left(\begin{array}{cc}
\mathbf{d} & -\overline{\mathbf{c}} \\
\mathbf{b} & \overline{\mathbf{a}}
\end{array}\right)\left(\begin{array}{cc}
1 & 0 \\
0 & -1
\end{array}\right)=\left(\begin{array}{cc}
\mathbf{b} & -\overline{\mathbf{a}} \\
-\mathbf{d} & -\overline{\mathbf{c}}
\end{array}\right) .
\end{gathered}
$$

Therefore, the improved equation is equivalent to:

$$
\begin{aligned}
& \left(E+\frac{\alpha}{r}\right)\left(\begin{array}{cc}
i \mathbf{d} & i \overline{\mathbf{c}} \\
i \mathbf{b} & -i \overline{\mathbf{a}}
\end{array}\right)+\left(\begin{array}{cc}
\partial_{r} \mathbf{d} & -\partial_{r} \overline{\mathbf{c}} \\
-\partial_{r} \mathbf{b} & -\partial_{r} \overline{\mathbf{a}}
\end{array}\right)+\frac{1}{r}\left(\begin{array}{cc}
\partial_{\theta} \mathbf{b} & \partial_{\theta} \overline{\mathbf{a}} \\
\partial_{\theta} \mathbf{d} & -\partial_{\theta} \overline{\mathbf{c}}
\end{array}\right) \\
& +\frac{\lambda}{r \sin \theta}\left(\begin{array}{cc}
\mathbf{b} & -\overline{\mathbf{a}} \\
-\mathbf{d} & -\overline{\mathbf{c}}
\end{array}\right)=i \mathrm{e}^{-i \beta}\left(\begin{array}{cc}
m \mathbf{a} & m \overline{\mathbf{b}} \\
m \mathbf{c} & -m \overline{\mathbf{d}}
\end{array}\right) .
\end{aligned}
$$

Conjugating the equations containing the terms of the right columns we get the system:

$$
\begin{aligned}
& i\left(E+\frac{\alpha}{r}\right) \mathbf{d}+\partial_{r} \mathbf{d}+\frac{1}{r}\left(\partial_{\theta}+\frac{\lambda}{\sin \theta}\right) \mathbf{b}=i m \mathrm{e}^{-i \beta} \mathbf{a}, \\
& -i\left(E+\frac{\alpha}{r}\right) \mathbf{c}-\partial_{r} \mathbf{c}+\frac{1}{r}\left(\partial_{\theta}-\frac{\lambda}{\sin \theta}\right) \mathbf{a}=-i m \mathrm{e}^{i \beta} \mathbf{b}, \\
& i\left(E+\frac{\alpha}{r}\right) \mathbf{b}-\partial_{r} \mathbf{b}+\frac{1}{r}\left(\partial_{\theta}-\frac{\lambda}{\sin \theta}\right) \mathbf{d}=i m \mathrm{e}^{-i \beta} \mathbf{c}, \\
& -i\left(E+\frac{\alpha}{r}\right) \mathbf{a}+\partial_{r} \mathbf{a}+\frac{1}{r}\left(\partial_{\theta}+\frac{\lambda}{\sin \theta}\right) \mathbf{c}=-i m \mathrm{e}^{i \beta} \mathbf{d} .
\end{aligned}
$$

Moreover we have:

$$
\rho \mathrm{e}^{i \beta}=\operatorname{det}(\phi)=\frac{\operatorname{det}(X)}{r^{2} \sin \theta}=\frac{\mathbf{a} \overline{\mathbf{d}}+\mathbf{c} \overline{\mathbf{b}}}{r^{2} \sin \theta}
$$

then we get: 


$$
\mathrm{e}^{i \beta}=\frac{\mathbf{a} \overline{\mathbf{d}}+\mathbf{c} \overline{\mathbf{b}}}{|\mathbf{a} \overline{\mathbf{d}}+\mathbf{c} \overline{\mathbf{b}}|} .
$$

In the Equation (53) only two angular operators are present, then we let:

$$
\mathbf{a}=A U ; \mathbf{b}=B V ; \mathbf{c}=C V ; \mathbf{d}=D U,
$$

where $A, B, C$ et $D$ are functions of $r$ while $U$ and $V$ are functions of $\theta$. The (53) system becomes:

$$
\begin{aligned}
& i\left(E+\frac{\alpha}{r}\right) D U+D^{\prime} U+\frac{1}{r}\left(V^{\prime}+\frac{\lambda}{\sin \theta} V\right) B=i m \mathrm{e}^{-i \beta} A U, \\
& -i\left(E+\frac{\alpha}{r}\right) C V-C^{\prime} V+\frac{1}{r}\left(U^{\prime}-\frac{\lambda}{\sin \theta} U\right) A=-i m \mathrm{e}^{i \beta} B V, \\
& i\left(E+\frac{\alpha}{r}\right) B V-B^{\prime} V+\frac{1}{r}\left(U^{\prime}-\frac{\lambda}{\sin \theta} U\right) D=i m \mathrm{e}^{-i \beta} C V, \\
& -i\left(E+\frac{\alpha}{r}\right) A U+A^{\prime} U+\frac{1}{r}\left(V^{\prime}+\frac{\lambda}{\sin \theta} V\right) C=-i m \mathrm{e}^{i \beta} D U .
\end{aligned}
$$

Then if a $\kappa$ constant exists such that:

$$
U^{\prime}-\frac{\lambda}{\sin \theta} U=-\kappa V ; V^{\prime}+\frac{\lambda}{\sin \theta} V=\kappa U,
$$

the (57) system becomes:

$$
\begin{aligned}
& i\left(E+\frac{\alpha}{r}\right) D+D^{\prime}+\frac{\kappa}{r} B=i m \mathrm{e}^{-i \beta} A \\
& -i\left(E+\frac{\alpha}{r}\right) C-C^{\prime}-\frac{\kappa}{r} A=-i m \mathrm{e}^{i \beta} B \\
& i\left(E+\frac{\alpha}{r}\right) B-B^{\prime}-\frac{\kappa}{r} D=i m \mathrm{e}^{-i \beta} C \\
& -i\left(E+\frac{\alpha}{r}\right) A+A^{\prime}+\frac{\kappa}{r} C=-i m \mathrm{e}^{i \beta} D
\end{aligned}
$$

To get the system equivalent to the Dirac equation it is enough to suppress the $\beta$ angle. This does not change the angular system (58), while in the place of (59) we get the system:

$$
\begin{aligned}
& i\left(E+\frac{\alpha}{r}\right) D+D^{\prime}+\frac{\kappa}{r} B=i m A, \\
& -i\left(E+\frac{\alpha}{r}\right) C-C^{\prime}-\frac{\kappa}{r} A=-i m B, \\
& i\left(E+\frac{\alpha}{r}\right) B-B^{\prime}-\frac{\kappa}{r} D=i m C, \\
& -i\left(E+\frac{\alpha}{r}\right) A+A^{\prime}+\frac{\kappa}{r} C=-i m D .
\end{aligned}
$$

\section{Kinetic Momentum Operators}

We established in [8] the form that the operators of kinetic momentum have in space-time. With the Pauli algebra we have (a detailed calculation is in [13] A.3): 


$$
\begin{gathered}
J_{1} \phi=\left(d_{1}+\frac{1}{2} \sigma_{23}\right) \phi \sigma_{21} ; d_{1}=x^{2} \partial_{3}-x^{3} \partial_{2}=-\sin \varphi \partial_{\theta}-\frac{\cos \varphi}{\tan \theta} \partial_{\varphi}, \\
J_{2} \phi=\left(d_{2}+\frac{1}{2} \sigma_{31}\right) \phi \sigma_{21} ; d_{2}=x^{3} \partial_{1}-x^{1} \partial_{3}=\cos \varphi \partial_{\theta}-\frac{\sin \varphi}{\tan \theta} \partial_{\varphi}, \\
J_{3} \phi=\left(d_{3}+\frac{1}{2} \sigma_{12}\right) \phi \sigma_{21} ; d_{3}=x^{1} \partial_{2}-x^{2} \partial_{1}=\partial_{\varphi} .
\end{gathered}
$$

We indeed have also:

$$
J^{2}=J_{1}^{2}+J_{2}^{2}+J_{3}^{2} .
$$

From (26) we have the following equivalence for $J_{3}$ :

$$
J_{3} \phi=\lambda \phi \Leftrightarrow \phi=\phi\left(x^{0}, r, \theta\right) \mathrm{e}^{\lambda \varphi i_{3}} .
$$

Then the $\phi$ wave satisfying (26) is a proper vector of $J_{3}$ and $\lambda$ is the magnetic quantum number. Moreover for a wave $\phi$ satisfying (26), we have:

$$
J^{2} \phi=j(j+1) \phi,
$$

if and only if:

$$
\partial_{\theta \theta}^{2} X+\left[\left(j+\frac{1}{2}\right)^{2}-\frac{\lambda^{2}}{\sin ^{2} \theta}\right] X-\lambda \frac{\cos \theta}{\sin ^{2} \theta} \sigma_{12} X \sigma_{12}=0 .
$$

And (58) implies at the second order:

$$
\begin{gathered}
0=U^{\prime \prime}+\left(\kappa^{2}-\frac{\lambda^{2}}{\sin ^{2} \theta}\right) U+\lambda \frac{\cos \theta}{\sin ^{2} \theta} U \\
0=V^{\prime \prime}+\left(\kappa^{2}-\frac{\lambda^{2}}{\sin ^{2} \theta}\right) V-\lambda \frac{\cos \theta}{\sin ^{2} \theta} V \\
0=\partial_{\theta \theta}^{2} X+\left(\kappa^{2}-\frac{\lambda^{2}}{\sin ^{2} \theta}\right) X-\lambda \frac{\cos \theta}{\sin ^{2} \theta} \sigma_{12} X \sigma_{12},
\end{gathered}
$$

therefore $\phi$ is a proper vector of $J^{2}$, with the proper value $j(j+1)$, if and only if:

$$
\kappa^{2}=\left(j+\frac{1}{2}\right)^{2} ;|\kappa|=j+\frac{1}{2} ; j=|\kappa|-\frac{1}{2} .
$$

with the definition of $S$ in (21) and with (26) we can see that the change of $\varphi$ into $\varphi+2 \pi$ conserves the same value for the wave if and only if $\lambda$ has a half-integer value. It is only in this case that the wave is correctly defined. The general results on the angular momentum operators imply then:

$$
j=\frac{1}{2}, \frac{3}{2}, \frac{5}{2}, \cdots ; \kappa= \pm 1, \pm 2, \pm 3, \cdots ; \lambda=-j,-j+1, \cdots j-1, j .
$$

To solve the angular system we let, if $\lambda>0$ and with $C=C(\theta)$ :

$$
\begin{aligned}
& U=\sin ^{\lambda} \theta\left[\sin \left(\frac{\theta}{2}\right) C^{\prime}-\left(\kappa+\frac{1}{2}-\lambda\right) \cos \left(\frac{\theta}{2}\right) C\right], \\
& V=\sin ^{\lambda} \theta\left[\cos \left(\frac{\theta}{2}\right) C^{\prime}+\left(\kappa+\frac{1}{2}-\lambda\right) \sin \left(\frac{\theta}{2}\right) C\right] .
\end{aligned}
$$


while if $\lambda<0$ we let:

$$
\begin{aligned}
& U=\sin ^{-\lambda} \theta\left[\cos \left(\frac{\theta}{2}\right) C^{\prime}+\left(\kappa+\frac{1}{2}+\lambda\right) \sin \left(\frac{\theta}{2}\right) C\right], \\
& V=\sin ^{-\lambda} \theta\left[-\sin \left(\frac{\theta}{2}\right) C^{\prime}+\left(\kappa+\frac{1}{2}+\lambda\right) \cos \left(\frac{\theta}{2}\right) C\right] .
\end{aligned}
$$

The angular system (58) is then equivalent [6] to the differential equation:

$$
0=C^{\prime \prime}+\frac{2|\lambda|}{\tan \theta} C^{\prime}+\left[\left(\kappa+\frac{1}{2}\right)^{2}-\lambda^{2}\right] C
$$

The change of variable:

$$
z=\cos \theta ; f(z)=C[\theta(z)]
$$

gives then the differential equation of the Gegenbauer polynomials ${ }^{1}$ :

$$
0=f^{\prime \prime}(z)-\frac{1+2|\lambda|}{1-z^{2}} z f^{\prime}(z)+\frac{\left(\kappa+\frac{1}{2}\right)^{2}-\lambda^{2}}{1-z^{2}} f(z) \text {. }
$$

And we get, as only integrable function:

$$
\frac{C(\theta)}{C(0)}=\sum_{n=0}^{\infty} \frac{\left(|\lambda|-\kappa-\frac{1}{2}\right)_{n}\left(|\lambda|+\kappa+\frac{1}{2}\right)_{n}}{\left(\frac{1}{2}+|\lambda|\right)_{n} n !} \sin ^{2 n}\left(\frac{\theta}{2}\right),
$$

with:

$$
(a)_{0}=1, \quad(a)_{n}=a(a+1) \cdots(a+n-1) .
$$

The $C(0)$ term is a factor of $U$ and $V$, its argument may be absorbed by the $\delta$ of (26), and its modulus may be transferred to the radial functions. We can then let $C(0)=1$, which gives:

$$
C(\theta)=\sum_{n=0}^{\infty} \frac{\left(|\lambda|-\kappa-\frac{1}{2}\right)_{n}\left(|\lambda|+\kappa+\frac{1}{2}\right)_{n}}{\left(\frac{1}{2}+|\lambda|\right)_{n} n !} \sin ^{2 n}\left(\frac{\theta}{2}\right) .
$$

Since we have the (71) conditions on $\lambda$ and $\kappa$, an integer $n$ always exists such as:

$$
|\lambda|+n=\left|\kappa+\frac{1}{2}\right| .
$$

This constrains the (80) series to be a finite sum, so then $U$ and $V$ are integrable. And since $U$ and $V$ have real values, we have:

$$
\mathrm{e}^{i \beta}=\frac{A \bar{D} U^{2}+C \bar{B} V^{2}}{\left|A \bar{D} U^{2}+C \bar{B} V^{2}\right|} .
$$

${ }^{1}$ When we solve the Dirac equation with Darwin's method, that means with the ad-hoc operators, we get some Legendre polynomials and spherical harmonics. Here, working with $\phi$, which is equivalent to working with the Weyl spinors $\xi$ and $\eta$, we get the Gegenbauer polynomials, and it is the degree of these polynomials that gives the needed quantum number. 


\section{Resolution of the Linear Radial System}

We employ the following transformations:

$$
\begin{aligned}
& x=m r ; \epsilon=\frac{E}{m} ; a(x)=A(r)=A\left(\frac{x}{m}\right), \\
& b(x)=B(r) ; c(x)=C(r) ; d(x)=D(r) .
\end{aligned}
$$

The (60) system becomes:

$$
\begin{aligned}
& i\left(\epsilon+\frac{\alpha}{x}\right) d+d^{\prime}+\frac{\kappa}{x} b=i a, \\
& -i\left(\epsilon+\frac{\alpha}{x}\right) c-c^{\prime}-\frac{\kappa}{x} a=-i b, \\
& i\left(\epsilon+\frac{\alpha}{x}\right) b-b^{\prime}-\frac{\kappa}{x} d=i c, \\
& -i\left(\epsilon+\frac{\alpha}{x}\right) a+a^{\prime}+\frac{\kappa}{x} c=-i d .
\end{aligned}
$$

And the (59) system becomes:

$$
\begin{aligned}
& i\left(\epsilon+\frac{\alpha}{x}\right) d+d^{\prime}+\frac{\kappa}{x} b=i \mathrm{e}^{-i \beta} a, \\
& -i\left(\epsilon+\frac{\alpha}{x}\right) c-c^{\prime}-\frac{\kappa}{x} a=-i b \mathrm{e}^{i \beta}, \\
& i\left(\epsilon+\frac{\alpha}{x}\right) b-b^{\prime}-\frac{\kappa}{x} d=i c \mathrm{e}^{-i \beta}, \\
& -i\left(\epsilon+\frac{\alpha}{x}\right) a+a^{\prime}+\frac{\kappa}{x} c=-i d \mathrm{e}^{i \beta} .
\end{aligned}
$$

Since this radial system has the same asymptotic behavior as its linear approximation (84) we are left with the following as the only integrable solution:

$$
\begin{aligned}
& a=\mathrm{e}^{-\Lambda x} x^{s}\left(a_{0}+a_{1} x+\cdots+a_{n} x^{n}\right) \\
& b=\mathrm{e}^{-\Lambda x} x^{s}\left(b_{0}+b_{1} x+\cdots+b_{n} x^{n}\right) \\
& c=\mathrm{e}^{-\Lambda x} x^{s}\left(c_{0}+c_{1} x+\cdots+c_{n} x^{n}\right) \\
& d=\mathrm{e}^{-\Lambda x} x^{s}\left(d_{0}+d_{1} x+\cdots+d_{n} x^{n}\right) ; \Lambda=\sqrt{1-\epsilon^{2}} .
\end{aligned}
$$

We now study the case where $n>0$. The (88) equation is equivalent to:

$$
\begin{aligned}
& i \epsilon\left(+a_{0} x+\cdots+a_{n-1} x^{n}+a_{n} x^{n+1}\right)+i \alpha\left(a_{0}+a_{1} x+\cdots+a_{n} x^{n}\right) \\
& +\Lambda\left(+a_{0} x+\cdots+a_{n-1} x^{n}+a_{n} x^{n+1}\right)-s\left(a_{0}+a_{1} x+\cdots+a_{n} x^{n}\right) \\
& -\left(+a_{1} x+\cdots+n a_{n} x^{n}\right)-\kappa\left(c_{0}+c_{1} x+\cdots+c_{n} x^{n}\right) \\
& =i \mathrm{e}^{i \beta}\left(+d_{0} x+\cdots+d_{n-1} x^{n}+d_{n} x^{n+1}\right) .
\end{aligned}
$$

Similarly, Equation (87) is equivalent to:

$$
\begin{aligned}
& i \epsilon\left(+b_{0} x+\cdots+b_{n-1} x^{n}+b_{n} x^{n+1}\right)+i \alpha\left(b_{0}+b_{1} x+\cdots+b_{n} x^{n}\right) \\
& +\Lambda\left(+b_{0} x+\cdots+b_{n-1} x^{n}+b_{n} x^{n+1}\right)-s\left(b_{0}+b_{1} x+\cdots+b_{n} x^{n}\right)
\end{aligned}
$$




$$
\begin{aligned}
& -\left(+b_{1} x+\cdots+n b_{n} x^{n}\right)-\kappa\left(d_{0}+d_{1} x+\cdots+d_{n} x^{n}\right) \\
& =i \mathrm{e}^{-i \beta}\left(+c_{0} x+\ldots+c_{n-1} x^{n}+c_{n} x^{n+1}\right) .
\end{aligned}
$$

Next, Equation (86) is equivalent to:

$$
\begin{aligned}
& i \epsilon\left(+c_{0} x+\cdots+c_{n-1} x^{n}+c_{n} x^{n+1}\right)+i \alpha\left(c_{0}+c_{1} x+\cdots+c_{n} x^{n}\right) \\
& -\Lambda\left(+c_{0} x+\cdots+c_{n-1} x^{n}+c_{n} x^{n+1}\right)+s\left(c_{0}+c_{1} x+\cdots+c_{n} x^{n}\right) \\
& +\left(+c_{1} x+\cdots+n c_{n} x^{n}\right)+\kappa\left(a_{0}+a_{1} x+\cdots+a_{n} x^{n}\right) \\
& =i \mathrm{e}^{i \beta}\left(+b_{0} x+\cdots+b_{n-1} x^{n}+b_{n} x^{n+1}\right) .
\end{aligned}
$$

Finally, Equation (85) is equivalent to:

$$
\begin{aligned}
& i \epsilon\left(+d_{0} x+\cdots+d_{n-1} x^{n}+d_{n} x^{n+1}\right)+i \alpha\left(d_{0}+d_{1} x+\cdots+d_{n} x^{n}\right) \\
& -\Lambda\left(+d_{0} x+\cdots+d_{n-1} x^{n}+d_{n} x^{n+1}\right)+s\left(d_{0}+d_{1} x+\cdots+d_{n} x^{n}\right) \\
& +\left(+d_{1} x+\cdots+n d_{n} x^{n}\right)+\kappa\left(b_{0}+b_{1} x+\cdots+b_{n} x^{n}\right) \\
& =i \mathrm{e}^{-i \beta}\left(+a_{0} x+\cdots+a_{n-1} x^{n}+a_{n} x^{n+1}\right) .
\end{aligned}
$$

We then arrive at three kinds of systems: index 0 , index between 0 and $n$, and index $n$. For the null index the system is independent of $\beta$ :

$$
\begin{aligned}
& (i \alpha-s) a_{0}-\kappa c_{0}=0 ;(i \alpha-s) b_{0}-\kappa d_{0}=0, \\
& \kappa a_{0}+(i \alpha+s) c_{0}=0 ; \kappa b_{0}+(i \alpha+s) d_{0}=0 .
\end{aligned}
$$

This system is the same as in the linear case. We obtain a non-null solution only if the determinant is null, then only if $s$ satisfies:

$$
0=\kappa^{2}+(i \alpha+s)(i \alpha-s) ; s=\sqrt{\kappa^{2}-\alpha^{2}} .
$$

And the system (94) is then equivalent to:

$$
c_{0}=\frac{i \alpha-s}{\kappa} a_{0} ; d_{0}=\frac{i \alpha-s}{\kappa} b_{0} .
$$

For the $n$ index the resulting system is:

$$
\begin{aligned}
& (i \epsilon+\Lambda) a_{n}=i \mathrm{e}^{i \beta} d_{n} ;(i \epsilon+\Lambda) b_{n}=i \mathrm{e}^{-i \beta} c_{n} ; \\
& (i \epsilon-\Lambda) d_{n}=i \mathrm{e}^{-i \beta} a_{n} ;(i \epsilon-\Lambda) c_{n}=i \mathrm{e}^{i \beta} b_{n} .
\end{aligned}
$$

From the definition of $\Lambda$ in (89) we obtain the cancellation of the determinants in the two previous sub-systems, and the (97) system is equivalent to:

$$
\begin{aligned}
& d_{n}=(\epsilon-i \Lambda) \mathrm{e}^{-i \beta} a_{n}, \\
& c_{n}=(\epsilon-i \Lambda) \mathrm{e}^{i \beta} b_{n} .
\end{aligned}
$$

Next the system with index between 0 and $n$ reads:

$$
\begin{aligned}
& (i \epsilon+\Lambda) a_{n-1}+(i \alpha-s-n) a_{n}-\kappa c_{n}=i \mathrm{e}^{i \beta} d_{n-1}, \\
& (i \epsilon-\Lambda) d_{n-1}+(i \alpha+s+n) d_{n}+\kappa b_{n}=i \mathrm{e}^{-i \beta} a_{n-1}, \\
& (i \epsilon+\Lambda) b_{n-1}+(i \alpha-s-n) b_{n}-\kappa d_{n}=i \mathrm{e}^{-i \beta} c_{n-1}, \\
& (i \epsilon-\Lambda) c_{n-1}+(i \alpha+s+n) c_{n}+\kappa a_{n}=i \mathrm{e}^{i \beta} b_{n-1} .
\end{aligned}
$$


Now we multiply (99) by $i \epsilon-\Lambda$ :

$$
(i \epsilon-\Lambda)(i \epsilon+\Lambda) a_{n-1}+(i \epsilon-\Lambda)(i \alpha-s-n) a_{n}-(i \epsilon-\Lambda) \kappa c_{n}=i \mathrm{e}^{i \beta}(i \epsilon-\Lambda) d_{n-1}
$$

Next (100) implies:

$$
(i \epsilon-\Lambda) d_{n-1}=i \mathrm{e}^{-i \beta} a_{n-1}-(i \alpha+s+n) d_{n}-\kappa b_{n} .
$$

And (103) becomes:

$$
\begin{gathered}
-a_{n-1}+(i \epsilon-\Lambda)(i \alpha-s-n) a_{n}-\kappa(i \epsilon-\Lambda) c_{n} \\
=i \mathrm{e}^{i \beta}\left[i \mathrm{e}^{-i \beta} a_{n-1}-(i \alpha+s+n) d_{n}-\kappa b_{n}\right] \\
(i \epsilon-\Lambda)(i \alpha-s-n) a_{n}-\kappa(i \epsilon-\Lambda) c_{n}=-i \mathrm{e}^{i \beta}(i \alpha+s+n) d_{n}-i \kappa \mathrm{e}^{i \beta} b_{n} .
\end{gathered}
$$

Using (97) we get:

$$
\begin{aligned}
& \mathrm{e}^{i \beta} d_{n}=(\epsilon-i \Lambda) a_{n}, \\
& \mathrm{e}^{i \beta} b_{n}=(\epsilon+i \Lambda) c_{n} .
\end{aligned}
$$

Substituting these relations into (105) we obtain:

$$
\begin{aligned}
& (\epsilon+i \Lambda)[-\alpha-i(s+n)] a_{n}-i \kappa(\epsilon+i \Lambda) c_{n} \\
& =-i(i \alpha+s+n)(\epsilon-i \Lambda) a_{n}-i \kappa(\epsilon+i \Lambda) c_{n} .
\end{aligned}
$$

Since $a_{n} \neq 0$ we get:

$$
\begin{gathered}
-(\epsilon+i \Lambda)[\alpha+i(s+n)]=[\alpha-i(s+n)](\epsilon-i \Lambda) \\
i \Lambda[\alpha-i(s+n)-\alpha-i(s+n)]=\epsilon[\alpha-i(s+n)+\alpha+i(s+n)], \\
\Lambda(s+n)=\epsilon \alpha .
\end{gathered}
$$

Squaring the equation and substituting the definition of $\Lambda$ we get:

$$
\begin{gathered}
\left(1-\epsilon^{2}\right)(s+n)^{2}=\epsilon^{2} \alpha^{2}, \\
(s+n)^{2}=\epsilon^{2}\left[\alpha^{2}+(s+n)^{2}\right], \\
\frac{E^{2}}{m^{2}}=\frac{(s+n)^{2}}{\alpha^{2}+(s+n)^{2}},
\end{gathered}
$$

which gives the Sommerfeld energy levels:

$$
E=\frac{m}{\sqrt{1+\frac{\alpha^{2}}{(s+n)^{2}}}} ; s=\sqrt{\kappa^{2}-\alpha^{2}} ;|\kappa|=j+\frac{1}{2} \in \mathbb{N}^{*} .
$$

\section{Constant Radial Polynomials}

To arrive at all the results of the Dirac equation, there is one last thing to explain: why we have $2 \mathbf{n}^{2}$ different bound states with principal quantum number $\mathbf{n}=|\kappa|+n$. For this we must return to the particular case where the radial polynomial is reduced to a constant. We begin directly from (84), and we let:

$$
a=a_{0} \mathrm{e}^{-\Lambda x} x^{s} ; b=b_{0} \mathrm{e}^{-\Lambda x} x^{s} ; c=c_{0} \mathrm{e}^{-\Lambda x} x^{s} ; d=d_{0} \mathrm{e}^{-\Lambda x} x^{s} .
$$


We obtain the following set of equations from (59):

$$
\begin{aligned}
& \mathrm{e}^{-\Lambda x}\left(i \epsilon d_{0} x^{s}+i \alpha d_{0} x^{s-1}-\Lambda d_{0} x^{s}+s d_{0} x^{s-1}+\kappa b_{0} x^{s-1}\right)=i a_{0} \mathrm{e}^{-\Lambda x} x^{s}, \\
& \mathrm{e}^{-\Lambda x}\left(-i \epsilon c_{0} x^{s}-i \alpha c_{0} x^{s-1}+\Lambda c_{0} x^{s}-s c_{0} x^{s-1}-\kappa a_{0} x^{s-1}\right)=-i b_{0} \mathrm{e}^{-\Lambda x} x^{s}, \\
& \mathrm{e}^{-\Lambda x}\left(i \epsilon b_{0} x^{s}+i \alpha b_{0} x^{s-1}+\Lambda b_{0} x^{s}-s b_{0} x^{s-1}-\kappa d_{0} x^{s-1}\right)=i c_{0} \mathrm{e}^{-\Lambda x} x^{s}, \\
& \mathrm{e}^{-\Lambda x}\left(-i \epsilon a_{0} x^{s}-i \alpha a_{0} x^{s-1}-\Lambda a_{0} x^{s}+s a_{0} x^{s-1}+\kappa c_{0} x^{s-1}\right)=-i d_{0} \mathrm{e}^{-\Lambda x} x^{s} .
\end{aligned}
$$

This is equivalent to the set formed by the four following systems:

$$
\begin{gathered}
\kappa b_{0}+(i \alpha+s) d_{0}=0, \\
(i \alpha-s) b_{0}-\kappa d_{0}=0, \\
-\kappa a_{0}-(i \alpha+s) c_{0}=0, \\
-(i \alpha-s) a_{0}+\kappa c_{0}=0, \\
-i a_{0}+(i \epsilon-\Lambda) d_{0}=0, \\
-(i \epsilon+\Lambda) a_{0}+i d_{0}=0, \\
i b_{0}-(i \epsilon-\Lambda) c_{0}=0, \\
(i \epsilon+\Lambda) b_{0}-i c_{0}=0 .
\end{gathered}
$$

The cancellation of the determinant in (117) and (118) gives again (95). The cancellation of the determinant in (117) and (118) is simply equivalent to $\Lambda^{2}=1-\epsilon^{2}$, which results from the definition of $\Lambda$. Each system (117) to (120) is then reduced to only one equation:

$$
\begin{aligned}
& \kappa d_{0}=(i \alpha-s) b_{0}, \\
& \kappa c_{0}=(i \alpha-s) a_{0}, \\
& d_{0}=(\epsilon-i \Lambda) a_{0}, \\
& b_{0}=(\epsilon+i \Lambda) c_{0} .
\end{aligned}
$$

We then obtain:

$$
\left.\kappa d_{0}=\kappa(\epsilon-i \Lambda) a_{0}=(i \alpha-s)\right)_{0}=(i \alpha-s)(\epsilon+i \Lambda) c_{0}=\frac{(i \alpha-s)^{2}(\epsilon+i \Lambda)}{\kappa} a_{0}
$$

We have a non-null solution only if:

$$
\begin{gathered}
\kappa(\epsilon-i \Lambda)=\frac{(i \alpha-s)^{2}(\epsilon+i \Lambda)}{\kappa}, \\
\kappa^{2}(\epsilon-i \Lambda)^{2}=(s-i \alpha)^{2} \\
\kappa(\epsilon-i \Lambda)= \pm(s-i \alpha) .
\end{gathered}
$$

Since $\epsilon, s, \Lambda$ and $\alpha$ are positive we finally get:

$$
|\kappa|=\frac{s}{\epsilon}=\frac{\alpha}{\Lambda} .
$$

This equality gives again the formula of the energy levels (114) with $n=0$. Since $\kappa$ comes with an absolute value, we can so well have $\kappa<0$ as $\kappa>0$. But Darwin's solutions, which work with real constants and not with complex 
ones at this stage of the calculation, forbid $\kappa$ to be negative and it is this interdiction which induces, for a principal quantum number $\mathbf{n}=n+|\kappa|$, a result of $\mathbf{n}(\mathbf{n}+1)+\mathbf{n}(\mathbf{n}-1)=2 \mathbf{n}^{2}$ states. This is what really happens: The change of sign of $\kappa$ is equivalent to changing $V$ into $-V$. And if we change the sign of $\kappa$ and of $V$, then $\mathbf{a}, \mathbf{b}, \mathbf{c}, \mathbf{d}$ are invariant if $n=0$, and the wave remains unchanged. Changing the sign of $\kappa$ brings no more supplementary solution and we can then use only the solutions with $\kappa>0$, in the case $n=0$. And this allows us to get the true number of states.

\section{Concluding Remarks}

Sommerfeld's formula (114) for energy levels does not account for the Lamb effect which is, if $n>0$, a very small shift between the energy levels with the same quantum numbers but with opposite values of $\kappa$. If the (114) formula was not the same for opposite values of $\kappa$, we should not be able to get four polynomial radial functions with only one condition which gives the quantification of the energy levels. The Standard Model has a precise answer using vacuum polarization. But the calculation must be revised, both to avoid divergences and to employ the improved wave equation which accounts also for weak interactions [33] [34]. Since the Lamb shift is of the same order as the hyperfine structure coming from the interaction between the magnetic moment of the proton and that of the electron, a true calculation must account for the origin of the magnetic moment of the proton from the waves of the three quarks inside, and for the true potential seen by the electron wave, with both the electric charges and the potential vector of the moving charges.

\section{Conflicts of Interest}

The authors declare no conflicts of interest regarding the publication of this paper.

\section{References}

[1] Lochak, G. (1983) Annales de la Fondation Louis de Broglie, 8, 4.

[2] Lochak, G. (1984) Annales de la Fondation Louis de Broglie, 9, 1.

[3] Lochak, G. (1985) International Journal of Theoretical Physics, 24, 1019-1050. https://doi.org/10.1007/BF00670815

[4] Hestenes, D. (1973) Journal of Mathematical Physics, 14, 893-905. https://doi.org/10.1063/1.1666413

[5] Hestenes, D. (1986) A Unified Language for Mathematics and Physics and Clifford Algebra and the Interpretation of Quantum Mechanics. In: Chisholm and Common, A., Eds., Clifford Algebras and Their Applications in Mathematics and Physics, Reidel, Dordrecht, 1-25, 321-346.

[6] Daviau, C. (1993) Equation de Dirac non linéaire. PhD Thesis, Université de Nantes, Nantes.

[7] Daviau, C. (1997) Advances in Applied Clifford Algebras, 7, 175-194.

[8] Daviau, C. (1997) Annales de la Fondation Louis de Broglie, 22, 87-103. 
[9] Daviau, C. (1998) Annales de la Fondation Louis de Broglie, 23, 1.

[10] Daviau, C. (2001) Annales de la Fondation Louis de Broglie, 26, 149-171.

[11] Daviau, C. (2005) Annales de la Fondation Louis de Broglie, 30, 3-4.

[12] Daviau, C. (2011) L'espace-temps double. JePublie, Pouillé-les-coteaux.

[13] Daviau, C. (2012) Double Space-Time and More. JePublie, Pouillé-les-coteaux.

[14] Daviau, C. (2012) Nonlinear Dirac Equation, Magnetic Monopoles and Double Space-Time. CISP, Cambridge.

[15] Daviau, C. (2012) Advances in Applied Clifford Algebras, 22, 611-623. https://doi.org/10.1007/s00006-012-0351-7

[16] Daviau, C. and Bertrand, J. (2012) Annales de la Fondation Louis de Broglie, 37, 129-134.

[17] Daviau, C. and Bertrand, J. (2014) New Insights in the Standard Model of Quantum Physics in Clifford Algebra. Je Publie, Pouillé-les-coteaux. http://hal.archives-ouvertes.fr/hal-00907848

[18] Daviau, C. and Bertrand, J. (2015) The Standard Model of Quantum Physics in Clifford Algebra. World Science Publishing, Singapore. https://doi.org/10.1142/9780

[19] Daviau, C. (2015) Advances in Applied Clifford Algebras, 27, 279-290. https://doi.org/10.1007/s00006-015-0566-5

[20] Daviau, C. (2013) Invariant Quantum Wave Equations and Double Space-Time. In: Advances in Imaging and Electron Physics, Vol. 179, Elsevier, Amsterdam, 1-137. https://doi.org/10.1016/B978-0-12-407700-3.00001-6

[21] Daviau, C. and Bertrand, J. (2014) JMP, 5, 1001-1022. https://doi.org/10.4236/jmp.2014.511102

[22] Daviau, C. and Bertrand, J. (2014) JMP, 5, 2149-2173. https://doi.org/10.4236/jmp.2014.518210

[23] Daviau, C. and Bertrand, J. (2015) Journal of Modern Physics, 6, 1647-1656. https://doi.org/10.4236/jmp.2015.611166

[24] Daviau, C. and Bertrand, J. (2015) Journal of Applied Mathematics and Physics, 3, 46-61. https://doi.org/10.4236/jamp.2015.31007

[25] Daviau, C. and Bertrand, J. (2015) Journal of Modern Physics, 6, 2080-2092. https://doi.org/10.4236/jmp.2015.614215

[26] Daviau, C. and Bertrand, J. (2015) Annales de la Fondation Louis de Broglie, 40, 181-209.

[27] Daviau, C. and Bertrand, J. (2016) Journal of Modern Physics, 7, 936-951. https://doi.org/10.4236/jmp.2016.79086

[28] Daviau, C., Bertrand, J. and Girardot, D. (2016) JMP, 7, 1568-1590. https://doi.org/10.4236/jmp.2016.712143

[29] Daviau, C., Bertrand, J. and Girardot, D. (2016) Journal of Modern Physics, 7, 2398-2417. https://doi.org/10.4236/jmp.2016.716207

[30] Daviau, C., Bertrand, J., Girardot, D. and Socroun, T. (2017) Annales de la Fondation Louis de Broglie, 42, 351-377.

[31] Daviau, C. and Bertrand, J. (2019) Journal of Modern Physics, 9, 250-258. https://doi.org/10.4236/jmp.2018.92017

[32] Daviau, C. and Bertrand, J. (2019) Annales de la Fondation Louis de Broglie, 44, 163-186. 
[33] Daviau, C., Bertrand, J., Socroun, T. and Girardot, D. (2019) Modèle standard et gravitation. Presses des Mines, Paris.

[34] Daviau, C., Bertrand, J., Socroun, T. and Girardot, D. (2020) Developing a Theory of Everything. Annales de la Fondation Louis de Broglie, Paris.

[35] Krüger, H. (1991) New Solutions of the Dirac Equation for Central Fields. In: Hestenes, D. and Weingartshofer, A., Eds., The Electron, Kluwer, Dordrecht, 49-81. https://doi.org/10.1007/978-94-011-3570-2_4 